\title{
Review of: "Disabling the Circadian Clock in the Yellow Fever Mosquito Aedes Aegypti Reduces Adult Life Span, Attraction to Host Odor and Mating Success"
}

\author{
Luciana O Araripe $^{1}$, Rafaela Bruno \\ 1 Fundação Oswaldo Cruz
}

Potential competing interests: The author(s) declared that no potential competing interests exist.

The article "Disabling the circadian clock in the yellow fever mosquito Aedes aegypti reduces adult life span, attraction to host odor and mating success" brings the effects of the putative knockout of cycle, one of the core clock genes, on aspects of Ae. aegypti behavior. The authors list negative impacts on the hatching rate, embryonic development and adult survival, on the mating success and on the response to host odors. On the other hand, cycle KO showed an increase in blood feeding propensity. Also, the authors highlight that knocking out cyc through CRISPR/Cas9 methodology caused alterations in the cyclical expression profiles of six of the seven clock genes studied, including cycle, as well as in the locomotor activity pattern of this species of mosquito.

While the construction of a clock-disrupted mosquito strain gives a longly desired tool for studying output physiological and behavioral traits, which are key parameters for the vector capacity and vector competence estimates, the current work lacks scientific rigor in methodological and interpretational elements. Also, literature citation was frequently inaccurate. Below we list minor and major problems that must be pointed out, with potential concerns on the validity of the article conclusions:

1) Mosquitoes are not disease vectors. Mosquitoes are vectors of pathogens that cause diseases.

2) Reference 11 is wrongly cited for "The endogenous circadian clock of mosquitoes regulates locomotor activity and blood feeding behavior". The cited article did not show a direct association between infection and the circadian clock, thus no regulation was implicated.

3) References 18-21 are wrongly cited for "the circadian clock also regulates the rhythmic expression of a broad range of genes that drive circadian behaviors". They were all published more than 20 years ago, before the use of high-throughput techniques for studying effects on a broad range of behavior genes.

4) Reference 27 is wrongly cited for "Cycle KO abolishes this peak in olfactory sensitivity, reducing overall sensitivity". The cited work did not use cycle KO, instead they measured circadian gene expression after a blood meal.

5) The same reference, 27, was wrongly cited again in "Female mosquitoes stop responding to host cues 
after blood-feeding, and it has been hypothesized that suppression of endogenous clock genes is the mechanism through which this is achieved". The cited studied did not test response to host cues.

6) How were the qRT-PCR results analyzed? The authors do not show the primers used to amplify gene SP6 and neither how they calculated the relative mRNA expression. Whether they used $\triangle C T$ or $\triangle \triangle C T$ method could enable or not comparisons among ZT collection points and between wildtype and AeCyc-/- mutants (Livak \& Schmittgen, 2001).

7) In "S2 cells were co-transfected with $5 \mathrm{ng} /$ well of pAC plasmids expressing wildtype AeClk, wildtype AeCyc and/or the mutant AeCyc", what is the portion of genes that is expressed in the plasmids?

8) Description of locomotor activity assays was quite superficial. How many individuals were monitored? Only inseminated females or males as well? How were the results averaged and plotted? See also what we wrote on (14).

9) Why 25-days old females were used to produce eggs for egg-hatching experiments? These may be quite old females, not commonly used for experiments intending to measure fitness parameters.

10) "To provide a human odor source, a white sock worn for two days by a volunteer was incubated at $370 C$ for three hours" - Is there a reference showing the efficacy of this method?

11) "Experiments conducted during the dark phase were conducted at $\sim 5$ lux". Previous work has shown that the circadian pacemaker responds to faint light, even 0.5 lux (Bachleitner W, Kempinger L, Wülbeck C, Rieger D, Helfrich-Förster C. Moonlight shifts the endogenous clock of Drosophila melanogaster. Proc Natl Acad Sci U S A. 2007;104(9):3538-3543. doi:10.1073/pnas.0606870104), which suggests that 5 lux may not be the ideal "darkness" for free run experiments.

12) "A total percentage of blood-fed AeCyc-/- females were compared with wildtype females at different time points." - The authors should indicate in the Methods section that comparisons were made in three categories, non-fed, partially fed and fully fed, as shown in the Results section (Table S3a).

13) "One-Way ANOVA and Post Hoc Bonferroni tests were used for pair-wise comparisons between AeCyc/- and wildtype at different time points during both LD and DD cycles." - Again, what values were used in these comparisons? $\triangle C T$ or $\Delta \triangle C T$ method? What values were used for normalization?

14) "Mosquito activity and host-seeking behavior in the wind tunnel were analyzed by generalized linear mixed models. Data were fit to a binomial distribution. Replicates were used as a random variable.

Genotype was used as the independent variable and the level of activity and responsiveness were used as the response variable." - This is not the usual method for analyzing locomotor activity. Taking into account that wild-type Ae. aegypti shows a bimodal pattern in LD conditions, as previously shown in a number of references, the adjustment to a pre-determined distribution could only generate a good fit in this specific setting. The authors do not show how much the other conditions (wild-type in DD, mutants in LD and DD) deviate from the predicted model, nor the parameters and goodness of fit of the GLM. Figure 4 only brings the average information; information on sample size and sample variation are lacking in the Figure and in the text.

15) "The mRNA expression study confirmed that the cyclical expression pattern of six clock genes is 
altered in AeCyc-/-" - from the described methodology, we cannot say whether gene expression is cycling or not. Usually expression values are compared among ZTs with ANOVA or circular statistics, and a significant P-value would suggest rhythmicity. Without these statistics, the authors cannot say a gene is showing a cyclical pattern. Thus, "Overall, the changed cyclical expression pattern of these genes points towards a disfunctional or disabled endogenous clock in AeCyc-/-" is not a valid conclusion.

16) All the life-history traits analyzed showed that the mutant AeCyc-/- is less fit than the wild type. This is an important result, but is also an expected outcome after the sylencing of a gene with a central regulating role, like cycle.

17) "Finally, although we did not quantify this measure, we note that AeCyc-/- females appeared to store less sperm, predominantly using only one or two of the three spermathecal capsules." - Sperm storage in fewer than three spermathecae is common is Aedes aegypti, and may not have to do with the silencing of cycle. See Carrasquilla et al. (2019) for a recent reference (Spermathecal Filling in Aedes aegypti and Aedes albopictus: Effects of Female and Male Body Sizes and Species).

18) "Interestingly, AeCyc-/- females are significantly less attracted to human odor than wildtype females at ZT12 and ZT18 ( $p=0.001$ and p=0.022, respectively, Figure 4C)." - An interesting result was the attraction of females to human odor in ZT18 (when both wildtype and mutant have almost no activity) in a level comparable to what was found in ZT6 and ZT12. The authors do not discuss why this might be.

19) "The blood feeding propensity of AeCyc-/- females peaked during daytime compared to night and somewhat surprisingly, was significantly $(p>0.005)$ higher than that of wildtype females at ZT1, ZT5, ZT13 and ZT17 (Figure 7, Table S3)" - The comparison shown in Figure 7 does not separate the level of bloodfeeding (non-fed, partially, fully), as in Table S3a. Was the comparison based solely on the fullyingurgitated females, as said in the last column of Table S3a? If so, why the values are different between Table S3a and Figure 7?

20) "Somewhat surprisingly, AeCyc-/- mRNA was detected in AeCyc-/- heads and did show a cyclical expression pattern." - In what position of the gene were the primers designed? If the primers were designed upstream of the deletion it is very possible that the detection of cycle expression is due to that. 21) "Consistent with a recent finding that PER protein levels are much reduced in circadian neuronal circuits of Ae. aegypti whose circadian clock is disrupted by exposure to constant light conditions[31], we did find a much reduced expression of per under both LD and DD conditions in heads of AeCyc-/-" - This sentence is nonsense to us. First, reference 31 is a Drosophila study and is cited for mosquitoes. Also, the authors extrapolate the finding that constant light conditions disrupt the endogenous clock to justify why reduced gene expression is observed in the KO individuals. The pathways for these effects may be completely different and associating the sources may lead to spurious conclusions.

22) "The significantly longer hatching time and lower hatching rates of AeCyc-/- indicates that this gene is involved in the proper timing and success of embryogenesis." - Mutations and knockouts often bring negative effects on development and fitness. This outcome may be direct or indirect, thus it does not indicate direct involvement in these functions. 
23) "A potential explanation could be the difference in chromosomal sex determination between

Drosophila and mosquitoes that Drosophila have heterogametic sex chromosomes, whereas Ae. aegypti do not." - We do not understand this rationale; if the authors see a relationship with the sexual system they should develop the idea deeper.

24) "This activity pattern indicates that light provides a strong stimulus and that the mosquito's response to this stimulus is strongly modulated by the clock." - This is not an indication coming from results of the current work, this is known since the first studies of circadian clock in insects.

25) "the lower sperm storage phenotype we observed in AeCyc-/- within an hour could be due to a delayed start in sperm production." - Are there previous studies observing this phenomenon? A more likely explanation could be that the KO males take longer to detect and seek females, delaying the number of matings and the sperm transfer, which would lead to fewer sperm in shorter intervals.

26) "Furthermore, per and tim expression was shown to be higher in swarming vs non- swarming fieldcollected mosquitoes." - What is the reference here?

27) "An. stephensi swarming behavior is strongest when per and tim expression peaks[48], which is not the case in Aedes aegypti, as shown in this study." - the current study did not analyze swarming behavior, thus the authors cannot make the comparison. "It is therefore not clear if the difference in expression level of these two genes could be responsible for the reduced mating success we observed." - why would this be related?

Overall, the article brings a compelling research question and a potential to generate valuable information for strategies of mosquito control. However, it is not clear how some of the data were analyzed and a new look on this should be recommended. Also, several results are discussed in a shallow and conjectural manner, resulting in potentially ambiguous conclusions. 